
- 



\title{
Mambrú vuelve a la escuela: politica, procesos y actores en América Latina
}

\author{
Manuel Franco-Avellaneda ${ }^{1}$ \\ mfrancoavellaneda@gmail.com \\ Oscar Alejandro Quintero R. ${ }^{2}$ \\ Universidad Nacional de Colombia, Bogotá, Colombia \\ oaquinteror@unal.edu.co \\ Recibido: $1{ }^{\circ}$ de febrero de 2016 \\ Aceptado: 13 de junio de 2016 \\ Disponible en línea: 20 de diciembre de 2016
}

\footnotetext{
Ingeniero Mecánico. Especialista en Pedagogía. Magíster en Educación. Doctor en Educación Científica y Tecnológica. Consultor en temas relacionados con educación científica y tecnológica y apropiación social de ciencia y tecnología (Bogotá, Colombia).

2 Sociólogo. Ph.D en Sociología. Profesor Asistente, Departamento de Sociología, Universidad Nacional de Colombia. Investigador, Grupo Interdisciplinario de Estudios de Género (GIEG), Centro de Estudios Sociales (CES) de la misma universidad (Bogotá, Colombia).
} 
Nunca antes en Colombia la educación estuvo tan presente en los medios de comunicación, ni en la agenda y el discurso públicos, circunstancia que se evidencia de variadas maneras. La política de gobierno respecto al tema es configurada por una dinámica problema-solución, en la que alcanzar la paz (problema) permitiría orientar esfuerzos en el cierre de brechas e invertir recursos en mejorar la cobertura y calidad del sistema educativo (solución) (DNP, 2015). En consecuencia, el Ministerio de Educación Nacional ha lanzado múltiples programas de créditos condonables en diferentes niveles: pregrado en Colombia y el exterior, y maestría para docentes. Además, se propone actualizar y subsanar buena parte del déficit de infraestructura física de escuelas en un tiempo récord de cuatros años, con 30 mil nuevos salones de clase. Sumado a lo anterior, se busca que la jornada escolar a la que concurren alrededor de 2'300.000 niños y niñas (más o menos una tercera parte del total de la matrícula) aumente de 5 o 6 horas -promedio actual-a 8 horas (OECD, 2016).

Asociado con lo anterior, universidades privadas que históricamente no contaban con formación de pregrado en licenciatura (formación inicial de docentes), ahora abren facultades y programas orientados a este nivel y tipo de formación. Así mismo, se reaviva una pugna entre un Estado laico y una escuela de fuerte influencia religiosa que se moviliza por cambios en los manuales de convivencia que reconocen en niños, niñas y jóvenes, orientaciones sexuales diversas, entre otras diferencias. Esta pugna, cabe decirlo, refleja tensiones más generales del conjunto de la sociedad.

Ese nuevo lugar que ocupa el Ministerio de Educación Nacional de Colombia entre las instituciones que más reciben recursos públicos, contrasta con los anuncios de Brasil de cerrar su programa "Ciência sem Fronteiras" y de hacer recortes en el presupuesto de las universidades federales de cerca del $50 \%$. En el caso de la educación básica y media, la disminución de recursos se evidencia en la franja infantil, pues se puso en crisis la meta de universalización en este nivel educativo, así como en el Programa Nacional de Acesso ao Ensino Técnico e Emprego (Mattos et al., 2016). Lo mismo sucede en Argentina, donde los recursos de las universidades se mantienen 
sin ajuste inflacionario, circunstancia que genera un pérdida de alrededor del $45 \%$ de los recursos de estas instituciones (Santucho-Ré, 2016), en tanto que en la educación básica se detienen programas como "Conectar igualdad", que ha distribuido computadores desarrollados localmente a más de cinco millones de niños, y los programas que atendian actividades extraescolares y fuera del currículo como radios escolares y orquestas infantiles y juveniles.

Ahora bien, estos contrastes entre lo que sucede en Colombia y otros países como Brasil y Argentina tienen un elemento común: las infraestructuras de funcionamiento del Estado se han mantenido casi iguales, y, por tanto, las políticas y sus programas asociados se abren o cierran acomodándose a ellas. Lo anterior genera una temporalidad de los procesos educativos asociada a los gobiernos de turno, que fácilmente se puede desmontar y re-priorizar dependiendo del cambio de intereses. Así las cosas, la educación como un campo en permanente transformación por razones endógenas y exógenas de los gobiernos, reconfigura los objetos de investigación y obliga a recrear aproximaciones ya exploradas en décadas pasadas.

En ese sentido, el presente número presenta una mirada compleja y variada de estudios sobre la educación en América Latina. Con base en investigaciones desarrolladas desde diversas disciplinas y abordajes conceptuales y metodológicos, los textos aquí reunidos permiten apreciar un panorama del estado de la cuestión en nuestro continente. Se trata de nueve artículos que abordan problemáticas de países como Argentina, Brasil y Colombia, que están llenos de aprendizajes, así como de nuevas preguntas y perspectivas por desarrollar en futuras investigaciones.

El artículo de Ana Maria Bartolini, Daniela Roxana Vivas, Carmela Ferreira y Natalia Petric, "Relatos y metáforas. Dos aproximaciones para comprender los procesos de tesis", presenta los hallazgos de una investigación que se inscribe en la línea de estudios que buscan comprender los complejos procesos de tesis de grado universitario, en este caso de egresados de dos facultades de la Universidad Autónoma de Entre Ríos (UADER), en Argentina. Para ello, sus autoras emplean dos 
fuentes de información: entrevistas focalizadas y selección de objetos (pintura, imagen, palabra, frase, foto, etc.), entendidas como fuentes discursivas y metafóricas, cuya confluencia enriquece y completa la comprensión sobre el proceso de tesis de los estudiantes participantes en la investigación.

Los principales hallazgos del estudio se fundamentan en la construcción de cuatro trayectorias grupales, en relación con el recorrido hecho por los estudiantes desde el inicio del proceso de tesis hasta el momento en que consideraron que finalizó. Este análisis de las trayectorias se complementa con un análisis de los objetos brindados por los mismos estudiantes como metáforas vinculadas con su proceso de tesis. El estudio de estas, muestra un alto grado de coherencia con el relato de los participantes. Dicho de otro modo, aparecen coincidencias o continuidades entre las expresiones discursivas y metafóricas por parte de los egresados, especialmente en relación con las "marcas" positivas o negativas que los diferentes momentos y protagonistas de los procesos les imprimieron.

En una perspectiva similar, relacionada con los procesos de violencia simbólica desarrollados en el campo educativo, se encuentra el artículo de Ana María Bartolini y Graciela Gerlo, "Reflexiones sobre experiencias de abandono de escuela secundaria y doctorado en Argentina. Modos de cierre y condicionantes individuales e institucionales". Este reflexiona críticamente sobre el abandono educativo, comparando las experiencias de desertores de escuelas secundarias y de programas doctorales en la República Argentina. Los resultados describen habitus auto-obstaculizadores de los desertores, modos de cierre del abandono y condicionantes institucionales. Se concluye, así, que el abandono educativo no es un episodio aislado, sino un momento dentro de un proceso que articula varias intervenciones, así como una sumatoria de condiciones y acontecimientos diversos, y que existe un "desenganche" progresivo que se produce tras frustraciones varias; paulatinamente, los estudiantes dejan de intentarlo, de interesarse, de esperar. El abandono llega cuando las energías son puestas definitivamente en función de otras metas. 
Por otro lado, muestran las autoras, el abandono está invisibilizado; el sistema educativo lo niega, lo encubre, lo minimiza, no quiere verlo. Prueba de ello son la inexistencia de listados de los desertores, así como la escasez de investigaciones y de políticas de retención estudiantil. El abandono es, predominantemente, producto de la exclusión académica. La reflexión a partir de los casos analizados lleva a la conclusión de que esta predominó, mientras que la deserción voluntaria fue excepcionalmente hallada. En todos los casos estudiados, tanto de secundaria como de doctorado, los entrevistados desplegaron las estrategias que estaban a su alcance, incluso, intentar culminar sus estudios durante varios años a pesar de obstáculos institucionales, de postergaciones personales y de efectos nocivos en la salud mental; por lo tanto, estas instituciones carecen del "ethos de cuidado" necesario para resolver las necesidades de los estudiantes.

Desde una perspectiva de análisis en torno a la tensión entre homogeneización y heterogeneidad del sistema, el artículo de Luanda Sito y Angela Kleiman, “'Eso no es lo mío': un análisis de conflictos en la apropiación de prácticas de literacidad académica", examina los conflictos que emergieron durante la apropiación de prácticas de literacidad académica de universitarios afros e indígenas que ingresaron en universidades públicas por acciones afirmativas en Brasil y Colombia.

El artículo identifica la forma como estos estudiantes vivencian conflictos en el proceso de apropiación de prácticas de literacidad académica, ya que necesitan lidiar con prácticas institucionales veladas, plenas de misterios, de la universidad. Igualmente, muestra cómo estos estudiantes crean estrategias para subvertir sentimientos de miedo y extrañeza, debido a la poca familiaridad - "eso no es lo mío"-, y pasar a sentirse parte de la universidad -a "revivir"-.

Al sumar un enfoque de la lingüística aplicada en la discusión sobre políticas públicas y sistemas educativos en el sur global, como las acciones afirmativas, las autoras llaman la atención sobre la importancia de comprender más íntimamente las experiencias de los sujetos que vivencian la implementación de esas políticas. Así, encuentran que los conflictos en las trayectorias de literacidad académica de esos 
estudiantes, además de denotar (des)encuentros con la universidad, interpelan a las políticas interculturales desde sus experiencias, revelando aspectos de la vida universitaria que no suelen ser contemplados en las discusiones de permanencia de los programas de acción afirmativa, como la interculturalidad y el lenguaje.

El artículo "Llevar La palabra. Un análisis de la relación entre las iglesias y la escolarización de niños indígenas tobas/qom y mbya-guaraní de Argentina", de Noelia Enriz, Mariana García Palacios y Ana Carolina Hecht, también se aproxima a la temática de minorias étnicas en la educación. El texto aborda el problema de la escolarización estatal, que no recuperó los conocimientos y experiencias de poblaciones indigenas en las que diversas iglesias persiguieron objetivos propios y reemplazaron, cuestionaron y apoyaron tareas del Estado. Así mismo, analiza los vínculos entre las iglesias (católica y evangélicas) y las poblaciones indígenas, específicamente en los ámbitos educativos y el proceso de alfabetización.

En ese sentido, el artículo identifica una multiplicidad de acciones llevadas a cabo, como la búsqueda de caminos pedagógicos y el interés por conocer la lengua y cultura de los indígenas en el marco de los programas evangelizadores y, especialmente, la inclusión de indígenas en estas tareas. Así, se muestra cómo las actuales políticas educativas mantienen un fuerte énfasis en la niñez, como momento formativo privilegiado a la hora de producir un acercamiento a aspectos culturales considerados como "otros": otra lengua, otra religión, otras formas de expresión y comunicación.

A su vez, las relaciones que las poblaciones indígenas desarrollaron y desarrollan con diversas agencias representan un ámbito sugerente para reflexionar en torno a los efectos de Estado, entendidos como instancias en las que los intereses estatales son representados o llevados a cabo por otros actores. En primer lugar, resulta interesante tener en cuenta que las normas locales que amparan a los grupos indígenas se anclan en una normativa internacional, que es considerada de mayor rango, el Convenio 169 de la OIT. Finalmente, la relación de estas poblaciones con el Estado se ve atravesada, además, por un 
internacionalismo que las vincula fuertemente a otros países del continente, pues sus organizaciones y contactos anteceden y trascienden los límites territoriales impuestos por el Estado.

En este estudio se evidencia el rol activo de las poblaciones indígenas en un proceso que en algunos casos dio origen a nuevas iglesias, y en otros mostró el rechazo por algunos credos o la falta de permeabilidad de las ideas religiosas en ciertos grupos. Así mismo, quedó claro que los procesos evangelizadores pudieron ser rechazados por ciertos grupos, por vía del éxodo o bien por la indiferencia. Esta asociación diferencial entre grupos y religiones/iglesias específicas evidencia el rol de las poblaciones indígenas frente a las misiones y sus posibilidades de acción.

En unos y otros casos, el artículo muestra cómo los ámbitos formativos que las propias comunidades utilizan para sí mismas se han mantenido como una incógnita. Las propuestas no han recabado nunca en los ámbitos existentes, que los propios grupos utilizan para dinamizar su cultura cotidianamente, generando nuevos espacios y modalidades de intercambio. La brecha entre unos y otros permitió a algunos miembros de las comunidades discurrir por ambos carriles, en un imbricado proceso de identificación.

Por su parte, el artículo de Héctor Andrés Pérez Enciso, "Problemas, actores y decisiones en las politicas públicas. Marco de análisis para el estudio de los programas de crédito educativo en Colombia", propone una perspectiva analítica para el estudio de los procesos de decisión asociados a la implementación de los créditos educativos en Colombia, como dispositivo para el fomento del acceso a la educación superior. Para ello, el autor hace un análisis de relaciones entre actores, problemas y decisiones políticas. Apoyándose en la teoría general de sistemas, Pérez Enciso propone un estudio en tres niveles: en una primera instancia, comprender cómo se configura el problema público que da origen a los créditos como dispositivo de política para fomentar el acceso a la educación superior. Una vez hecho el análisis del problema, el artículo se orienta a identificar los actores inmersos en la arena de la política educativa y el lugar desde el cual se enuncian sus propuestas. Finalmente, aborda las decisiones 
que llevan a la escogencia de una estrategia o curso de acción de un abanico más amplio de posibilidades. Este artículo es un insumo importante en la comprensión del "boom" de créditos educativos promovidos por la política educativa del gobierno actual en Colombia, que se ha orientado a estimular la demanda para fortalecer la oferta, circunstancia que ha generado una multiplicación exponencial de cupos, y una consecuente ampliación de las universidades, principalmente privadas, en docentes e infraestructura fisica para captar los recursos que representan los beneficiarios de los créditos.

Asociado con la toma de decisiones en la administración pública, el artículo "Políticas educativas en Latinoamérica: la vinculación entre los investigadores académicos y tomadores de decisiones en educación. Un análisis desde la teoría de los campos”, de César Tello, propone abordar este tópico a partir de un análisis desde la perspectiva de "campos", para comprender las relaciones entre los actores sociales y su rol en la red sociopolítica. Desde ahí, el autor identifica que la producción de conocimiento y la toma de decisiones requieren de espacios para intercambiar, dialogar, proyectar. En casos en los que estos espacios no existen se impide que el conocimiento pueda entrar a las esferas de los tomadores de decisiones y ayudar a los gestores en la arena política. En esa línea, el artículo propone un interesante análisis de dos campos, tomadores de decisión e investigadores, el cual puede servir de base para responder preguntas asociadas a las lógicas políticas del contexto latinoamericano, tales como: ¿qué investigadores y centros tienen voz y participación en las esferas políticas?, ¿cómo opera el capital cultural de los investigadores en relación al "uso y aprovechamiento" de sus investigaciones? y ¿cómo se configuran problemas y soluciones en el ámbito de la esfera de tomadores de decisión en los temas educativos?

Por su parte, Claudia Milena Díaz Ríos, en su artículo "La traducción de las ideas globales en la gobernanza de la educación secundaria en Colombia", muestra cómo las reformas educativas en Colombia siguen una tendencia global en la que coinciden la descentralización curricular y la privatización de la educación, y que dichas tendencias sufren procesos de traducción desde los que se acomodan prácticas 
domésticas. En ese sentido, la autora discute las teorias de la difusión que suponen una cierta "copia" de las ideas y muestra qué características tiene la traducción de ideas educativas globales en Colombia, desde la lógica de los mediadores (aquellos que tienen contacto con las ideas globales y con los escenarios locales), quienes transforman, traducen, distorsionan y modifican el significado o los elementos que se supone deben implementar. Esto reafirma los planteamientos de la teoria social de las últimas décadas, que reconoce que las ideas se recrean localmente en una lógica de apropiación, lo cual invita permanentemente a investigar cómo suceden los ensamblajes locales de ideas globales.

Ahora bien, en relación a cómo operan los procesos educativos a escala local, hay dos artículos que abordan desde dos puntos de vista diferenciados los procesos en el sistema educativo. De un lado, el artículo de Luisa Iñigo y Victoria Rio, "Extensión de la escolaridad y obligatoriedad de la escuela secundaria en Argentina: el papel de la universalización de la lectura y escritura", muestra cómo la obligatoriedad escolar tiene una premisa asociada a la lectoescritura. Además, expone cómo el aprendizaje de esas habilidades tiene una correspondencia evidente con una adquisición diferenciada de capacidades lectoras y escritoras por región geográfica en Argentina. Así, los desempeños parecen reflejar el lugar ocupado por los estudiantes y sus familias en la estructura social. Esto corrobora que en Latinoamérica las oportunidades educativas, tanto en acceso como en calidad, aún están condicionadas por el origen socioeconómico, la ubicación geográfica, la etnia y el género.

De otro lado, en el artículo de Rocío Rueda, "Subjetividad y tecnologías de la escritura. La formación en tiempos de poshumanismo", se describen los mecanismos de co-formación en la plataforma Facebook: programabilidad, popularidad, conectividad y dataficación, donde concurren diferentes agencias de humanos y no humanos que configuran procesos informales de formación individual y colectiva. Este texto pone sobre la mesa, con otras herramientas de análisis provenientes de los estudios sociales de ciencia y tecnología, la ya vieja discusión sobre la pérdida de hegemonía de la escuela como lugar para la adquisición de 
contenidos. En efecto, cinco décadas atrás, un niño al dejar la escuela quedaba por fuera de la influencia de la lectoescritura. Por el contrario, en la actualidad, un niño al salir de la escuela queda siempre "atrapado" en las redes comunicacionales en las que suceden también procesos educativos.

Este número de la revista Universitas Humanística nos muestra un panorama diverso sobre la educación y sus problemas sociales contemporáneos. Se identifican al menos tres elementos centrales en relación con esta temática que se constituyen en desafios para los estudiosos del tema, pero también para los sectores públicos y privados que influyen en la toma de decisiones del sector. En primer lugar, un campo temático relevante es el de las políticas públicas en la globalización, las tensiones entre agendas internacionales, nacionales y locales, en el que entran a jugar un rol importante las dinámicas de los distintos actores y sus mediaciones en la construcción de las políticas públicas.

En segundo lugar, se muestra la vigencia de los estudios sobre la educación como un campo marcado por violencias simbólicas, por la tensión entre homogeneización de poblaciones que son heterogéneas. En ese sentido, es crucial el estudio de las formas de adaptación o no a esas nuevas poblaciones que logran acceder al derecho educativo por diversas dinámicas sociales, algunas de estas coyunturales, como sería el caso de las personas desmovilizadas de grupos armados en Colombia. Así mismo, el desafio de la educación consiste aun más que nunca en constituirse en una vía legítima, aceptada e incontestable para la movilidad social de poblaciones históricamente marginadas.

Por último, algunos de los estudios aquí presentados abren nuevas preguntas en relación con la educación como lógica de humanización. Si bien la educación está asociada con procesos de (re)producción de desigualdades sociales, esta también se convierte en un elemento central de democratización de oportunidades en las sociedades contemporáneas, generación de condiciones para el ejercicio de la ciudadanía y de emancipación individual y colectiva. Quizás esto último sea el gran desafío no solo para quienes investigamos la educación, sino 
para las sociedades latinoamericanas que desean que Mambrú vuelva a la escuela.

\section{Referencias}

DNP. (2015). Plan Nacional de Desarrollo 2014-2018. Todos por un nuevo país. Bogotá D.C.: Departamento Nacional de Planeación - República de Colombia.

Mattos, R. da S., Espírito-Santo, W., Castro, J. B. P. de, Retondar, J. M., Oliveira, L. H. y Nascimento, S. (2016). O futuro do pretérito na educação brasileira: declinações dos direitos sociais sob a égide de "uma ponte para o futuro". Revista Brasileira de Sociologia Do Direito, 3(1), 171-193.

OECD. (2016). Reviews of National Policies for Education. Education in Colombia. Paris, France: OECD.

Santucho-Ré, N. (2016). Universidad: hegemonía liberal y nuevos desafíos. Letras-Centro de Investigación en Lectura y Escritura (CILE), 89-95.

\section{Cómo citar este artículo}

Franco-Avellaneda, M. y Quintero R., O. A. (2017). Mambrú vuelve a la escuela: política, procesos y actores en América Latina. Universitas Humanística, 83,19-29. http://dx.doi.org/10.11144/Javeriana.uh83.mvep 